\title{
Patient Characteristics and Risk Factors in Invasive Mold Infections: Comparison from a Systematic Review and Database Analysis
}

\author{
Anita H Sung' \\ Stephan Martin ${ }^{2}$ \\ Bryant Phan (ID) \\ Michael Benigno (D) \\ Jennifer Stephens ${ }^{2}$ \\ Richard Chambers' \\ Jalal A Aram' \\ 'Pfizer Inc, New York, NY, USA; ${ }^{2}$ OPEN \\ Health, Bethesda, MD, USA
}

\begin{abstract}
Introduction: Diagnosis and treatment of invasive mold infections (IMI) can be challenging and IMI is a significant source of morbidity and mortality. Invasive aspergillosis (IA) and invasive mucormycosis (IM) are two of the most common mold infections. A better understanding of patient comorbidities and risk factors that predispose IMI may help clinicians to refine the difficult diagnostic and treatment process.
\end{abstract}

Methods: A systematic literature review (SLR) was conducted (January 2008October 2019) for studies reporting comorbidities/risk factors of patients with IA or IM (Phase I), followed by an analysis on the Optum ${ }^{\circledR}$ US EHR database of prominent risk factor cohorts based on SLR findings and expert opinion (Phase II). From the four identified patient cohorts: 1) patients undergoing solid organ transplant (SOT) and patients with 2) hematologic cancers, 3) diabetes, or 4) lung disease, rates of IA, IM, or concurrent IA and IM; patient comorbidities; and Charlson Comorbidity Index (CCI) scores were reported.

Results: The SLR included 88 studies, and 46 were used to select comorbidities/risk factors cohorts in IA and IM patients. The most important comorbidities/risk factors in IA and IM patients were diabetes, lung disease, hematological malignances, and SOT. In the Optum database $(\mathrm{N}=101,340,454$ patients), IA rates were highest in lung transplant $(10.81 \%)$ patients and IM rates were highest in intestine transplant $(0.83 \%)$ patients, lung transplant $(0.43 \%)$, and hematopoietic stem cell transplant $(0.49 \%)$. CCI scores were elevated in all mold infection groups compared to the total Optum cohort.

Conclusion: The current study describes patient comorbidity and risk factors associated with IA and IM. These data can be used to refine clinical decision-making regarding when to suspect mold infections. Future research should focus on identifying whether patients respond differently to various antifungal treatments to determine if strategic recommendations should be made for certain patient groups.

Keywords: invasive mold infections, systematic literature review, retrospective claims data, invasive aspergillosis, invasive mucormycosis

\section{Introduction}

Invasive mold infections are a growing problem worldwide with invasive aspergillosis (IA) and invasive mucormycosis (IM) as two of the most common mold infections. $^{1-4}$ Increasing incidence of IA and IM is multifactorial. More widespread adoption of aggressive therapy practices including chemotherapy, transplantation, and intensive care use contributes to greater numbers of infections. ${ }^{5}$ Also, there are emerging mold infection populations such as Influenza- and SARS-CoV-
Correspondence: Anita $\mathrm{H}$ Sung Pfizer, 235 E 42nd St, New York, NY, 10017 , USA

Tel +l 212 733-2323

Email anita.sung@pfizer.com 
2-associated Aspergillosis, ${ }^{6,7}$ as well as mold infections in patients with B cell cancers treated with ibrutinib. ${ }^{8}$ Despite advances in clinical understanding, invasive mold infections remain a significant source of morbidity and mortality. ${ }^{9}$ If untreated, mortality in patients diagnosed with invasive aspergillosis up to nearly $85 \%$ depending on the underlying condition, ${ }^{10,11}$ and mortality in untreated patients with invasive mucormycosis is near $100 \%{ }^{12,13}$

Epidemiology of invasive mold infections varies between hospitals and regions of the world and is influenced by population risk factors and antifungal use. ${ }^{5}$ For example, IM is rare, but increasingly prevalent globally in patients with uncontrolled diabetes mellitus. ${ }^{14}$ A high proportion of IM cases are diagnosed in the intensive care unit (ICU). ${ }^{14}$ Risk factors for both IA and IM have been extensively studied in patients with hematologic malignancy and transplants, however, clinical and patient risk factors are less well defined for non-traditional and emerging at-risk patient groups. ${ }^{15}$ The advent of new technology such as $18 \mathrm{~S}$ ribosomal RNA PCR and genome sequencing is leading to improved understanding of epidemiology of invasive mold infections. ${ }^{5}$

Diagnosis of invasive mold infections can be a challenge and mold is oftentimes not suspected. ${ }^{14}$ Detection of mold infections is difficult because 1) definitive diagnosis frequently requires tissue sampling by invasive procedure, ${ }^{16}$ 2) immune-compromised patients may be unable to mount an effective immune response, which affects antibody-based testing, ${ }^{17}$ and 3) many molds including IA are rarely isolated from blood cultures. ${ }^{18}$ The complexity of the diagnostic work-up, in which the clinical, radiological, and microbiological findings must be considered, contributes to a low detection rate for invasive mold infections. ${ }^{19-21}$ Early diagnosis and timely therapeutic intervention require a high level of awareness and suspicion.

Currently, available antifungal treatments target most mycoses encountered in clinical practice. ${ }^{22,23}$ However, significant differences exist between treatments regarding efficacy, toxicity profile, pharmacokinetics, formulation, and interactions with concomitant medications. ${ }^{23}$ Amphotericin B, released in the 1950s, remains one of the widest spectrum antifungal agents available. ${ }^{24}$ However, amphotericin B nephrotoxicity and requirement for intravenous administration are limiting. Voriconazole and the echinocandins are active against IA, yet lack meaningful or clinically proven activity against IM. $^{22}$ Isavuconazole is an example of one of a few recently approved triazoles with activity against both IA and IM. $^{25}$ In order to optimize antifungal treatments to unique patients, clinicians should understand not only properties of different antifungal agents but also patient comorbidities and risk factors in invasive mold infections.

A better understanding of the population at risk for invasive mold infections to inform suspicion of mold infection may contribute to improved outcomes for this potentially treatable disease. To this end, the objectives of this study were twofold. First, a systematic literature review (SLR) was conducted to determine risk factors and their rates of occurrence reported in the literature associated with IA, IM, or co-infection with both. Second, a descriptive analysis of a US insurance claims database was conducted to assess rates of IA and IM in patients with comorbidities and risk factors identified in the SLR.

\section{Materials and Methods Study Design}

This study was conducted in two phases. In the first phase (Phase I), a Systematic Literature Review (SLR) was conducted according to the Preferred Reporting Items for Systematic Reviews and Meta-Analyses (PRISMA). ${ }^{26}$ The SLR identified journal articles and conference abstracts reporting comorbidity/risk factors in patients with confirmed IA or IM. In the second phase (Phase II), a retrospective cohort analysis was performed on the Optum $^{\circledR}$ US EHR database on patient cohorts selected based on findings of the SLR and expert opinion. From these risk factor cohorts, patients with IA, IM, or concurrent IA and IM were identified. Rates of IA, IM or concurrent IA and IM; patient comorbidities; and Charlson Comorbidity Index (CCI) scores were reported.

\section{Phase I: Systematic Literature Review} Study Selection

Study inclusion and exclusion criteria are available in Table 1. Medline and EMBASE were searched for journal articles published in the time-period January 2008 to December 2019 (Appendix A). Conference abstracts from the European Hematology Association, American Society of Hematology, European Congress of Clinical Microbiology and Infectious Diseases, and Trends in Medical Mycology were searched for the time-period January 2015 to October 2019. All studies identified were reviewed independently by two reviewers for 
Table I Study Inclusion/Exclusion Criteria

\begin{tabular}{|c|c|c|}
\hline & Inclusion Criteria & Exclusion Criteria \\
\hline Population & - Adults (aged $\geq 18$ years) with IA or IM & $\begin{array}{l}\text { - Superficial/cutaneous or otherwise localized fun- } \\
\text { gal infections } \\
\text { - Children (aged } \leq 17 \text { years) with IMI }\end{array}$ \\
\hline Interventions & - Any treatment used in mold infection therapy & $\begin{array}{l}\text { - Prophylactic treatment with mold infection } \\
\text { treatment }\end{array}$ \\
\hline Comparators & - Any or none & - N/A \\
\hline Outcomes & - Risk factors and/or comorbidities associated with IMI & $\begin{array}{l}\text { - Non-included outcomes } \\
\text { - Outcomes not stratified by mold vs yeast } \\
\text { infections }\end{array}$ \\
\hline$\underline{\text { Study design }}$ & $\begin{array}{l}\text { - Observational studies: Prospective or retrospective } \\
\text { - Database analyses/registries } \\
\text { - Clinical trials } \\
\text { - Professional society guidelines } \\
\text { - Systematic literature reviews/meta-analyses (for study identification purposes } \\
\text { only) }\end{array}$ & $\begin{array}{l}\text { - Observational Non-systematic reviews } \\
\text { - Case series/case reports } \\
\text { - Commentary/editorial letter }\end{array}$ \\
\hline Setting & - Inpatient and outpatient & - N/A \\
\hline $\begin{array}{l}\text { Publication } \\
\text { type }\end{array}$ & $\begin{array}{l}\text { - Manuscripts published in medical or economic journals indexed in MEDLINE/ } \\
\text { - Cmbase } \\
\text { - Conference abstracts from } 4 \text { conferences: } \\
\text { - American Society of Hematology (ASH) } \\
\text { - Trends in Medical Mycology (TIMM) } \\
\text { - European Congress of Clinical Microbiology and Infectious Diseases (ECCMID) }\end{array}$ & - Non-included publication types \\
\hline Language & - English & - Non-English \\
\hline $\begin{array}{l}\text { Publication } \\
\text { date }\end{array}$ & $\begin{array}{l}\text { - Full-text articles: published January I, } 2008 \text { to October } 2019 \\
\text { - Conference abstracts: published January I, } 2015 \text { to October } 2019\end{array}$ & - Published prior to January I, 2008 \\
\hline Other & - Human subjects & - Preclinical/animal subjects \\
\hline
\end{tabular}

Abbreviations: IA, Invasive Aspergillosis; IM, Invasive Mucormycosis; IMI, Invasive mold infection; N/A, Not applicable.

selection. In cases where study selection was discordant, the researchers reviewed the article together to reach consensus. All studies included were reviewed by the responsible ethics committee during their original conduct, as such, there was no IRB approval required for this secondary data analysis.

\section{Data Extraction}

Information collected from each individual study included study design; data source; region of conduct (Africa and Middle East [AfME], The United States [US], Asia Pacific [APAC], Europe, Oceania, Canada, and Global [if spanning multiple regions]); inclusion and exclusion criteria; rates of comorbidities/risk factors present in IA and IM patients; and treatment details. Patient comorbidity/risk factors were grouped accordingly: Healthy, Diabetes,
Lung Disease, Liver Disease, Solid Organ Transplant, Hematology Transplant, Solid Malignancy, Hematology Malignancy or Disease, Heart Disease, Renal Impairment, Immunocompromised, and Others.

\section{Data Synthesis}

Data from studies that reported the comorbidities/risk factors for each mold cohort separately in the SLR were used to determine risk profiles of the different mold infection groups. The proportion of studies that mention each comorbidity/risk factor in IA or IM patients was assessed to determine which are most prevalent in the literature. Weighted average estimates of frequency of comorbidities/ risk factors in IA and IM patients were calculated by combining the frequency of comorbidities/risk factors in IA or IM patients reported in each individual study in the SLR. 


\section{Phase II: Database Analysis Data Source}

Optum's deidentified Integrated Claims-Clinical data set combines adjudicated claims data with electronic health record (EHR) data. The Optum ${ }^{\circledR}$ US EHR is derived from more than 50 healthcare provider organizations in the United States including more than 700 hospitals and 7000 clinics. All payment types are represented (commercial, Medicare, Medicaid, cash, and manufacturer's coupons). EHR information collected in the dataset includes medications prescribed and administered, laboratory results, vital signs, body measurements, diagnoses, and procedures. Optum ${ }^{\circledR}$ US EHR data were de-identified in compliance with the US Health Insurance Portability and Accountability Act (HIPAA). As such, there was no requirement for institutional review board/ethics approval.

\section{Inclusion Criteria}

A retrospective review was conducted of the Optum Integrated Claims-Clinical database from January 2007 to June 2019. Subjects were identified as having IA or IM using the following ICD-9 and ICD-10 codes: IA (ICD-9: 117.3, 117.9, 484.6, 518.6 or ICD-10: B44.0, B44.1, B44.2, B44.7, B44.81, B44.89, B44.9, B48.3, B48.4, B48.8, B49), IM (ICD-9: 117.7 or ICD-10: B46.x), or co-infection of both IA and IM. All ICD-9 and ICD-10 codes used in this study are available in the supplementary material Appendix C.

\section{IM and IA Comorbidity and Risk Factors}

ICD-9 and ICD-10 codes were used to identify presence of patient comorbidities/risk factors. The most prevalent risk factors from the literature were extracted and grouped using expert opinion. The most frequent patient comorbidities and the comorbidities that were cited the most in the literature were considered to select the following cohorts for the Optum ${ }^{\circledR}$ US EHR database analysis:

- diabetes,

- lung disease (including cystic fibrosis, tuberculosis, and chronic obstructive pulmonary disorder [COPD] diagnoses),

- hematology and oncology diagnoses (HEM/ONC; including acute myeloid leukemia [AML], lymphoma, leukemia, other, stem cell, and neutropenia diagnoses), and

- solid organ transplants (including kidney, heart, lung, liver, pancreas, heart and lung, pancreas and kidney, intestine, and other transplants).
Within these four patient risk cohorts, rates of IM, IA, or co-infection with both IA and IM were analyzed. In addition, frequencies of top ICD-9/ICD-10 codes within each risk cohort were used to assess additional comorbidities between the four main patient cohorts that may overlap in risk.

\section{Comorbidity Burden in Invasive Mold Infection Patients}

Charlson Comorbidity Index (CCI) scores were calculated for each patient. ${ }^{27,28}$ Pairwise comparisons of CCI score were conducted between each mold infection group (IA, IM, or co-infection with both) using a Student's $t$-test and a 0.05 level of statistical significance in each of the cohorts. CCI scores were interpreted as Mild (1-2), Moderate (3-5), and Severe (>5). ${ }^{27-29}$ Microsoft Excel (Microsoft Corp., Redmond, WA) and SAS version 9.4 (SAS Institute, Cary, North Carolina) was used to perform descriptive and comparative statistics.

\section{Results}

\section{Phase I Systematic Literature Review Summary}

2009 citations were screened. After full text review, 88 studies met inclusion criteria. Included studies reported on IA $(\mathrm{n}=52), \mathrm{IM}(\mathrm{n}=17)$, and co-infection with IA and IM $(n=18)$. Of these, 46 studies that reported only on risk factors associated with IA $(n=35)$ or IM $(n=11)$ were included in the final data synthesis, including 39 full-text articles and 7 conference abstracts (Appendix B). Studies were from AfME $(n=5)$, US $(n=8)$, APAC $(n=8)$, Europe $(n=15)$, Oceania $(n=1)$, Canada $(n=2)$, Global $(n=6)$, and not-specified $(\mathrm{n}=1)$. Study designs were retrospective $(n=34)$, prospective $(n=9)$, SLR $(n=4)$ studies, but some incorporated multiple study design components. The PRISMA flow chart is presented in Figure 1.

The proportion of IA and IM comorbidities/risk factors in the studies from the literature is reported in Table 2. Among the 35 IA studies identified in the SLR, the most prevalent comorbidities/risk factors were diabetes $(n=17)$, hematological malignancy $(\mathrm{n}=17)$, SOT $(\mathrm{n}=11)$, and lung disease $(n=10)$. Among the 11 IM studies identified in the SLR, the most prevalent comorbidities/risk factors were diabetes $(n=8)$, hematological malignancy $(n=7)$, and SOT $(\mathrm{n}=4)$, along with immunocompromised status $(\mathrm{n}=4)$.

The simple weighted average of prevalence of IA and IM patient comorbidities/risk factors from the literature is reported in Table 3. The most frequently occurring comorbidities/risk 


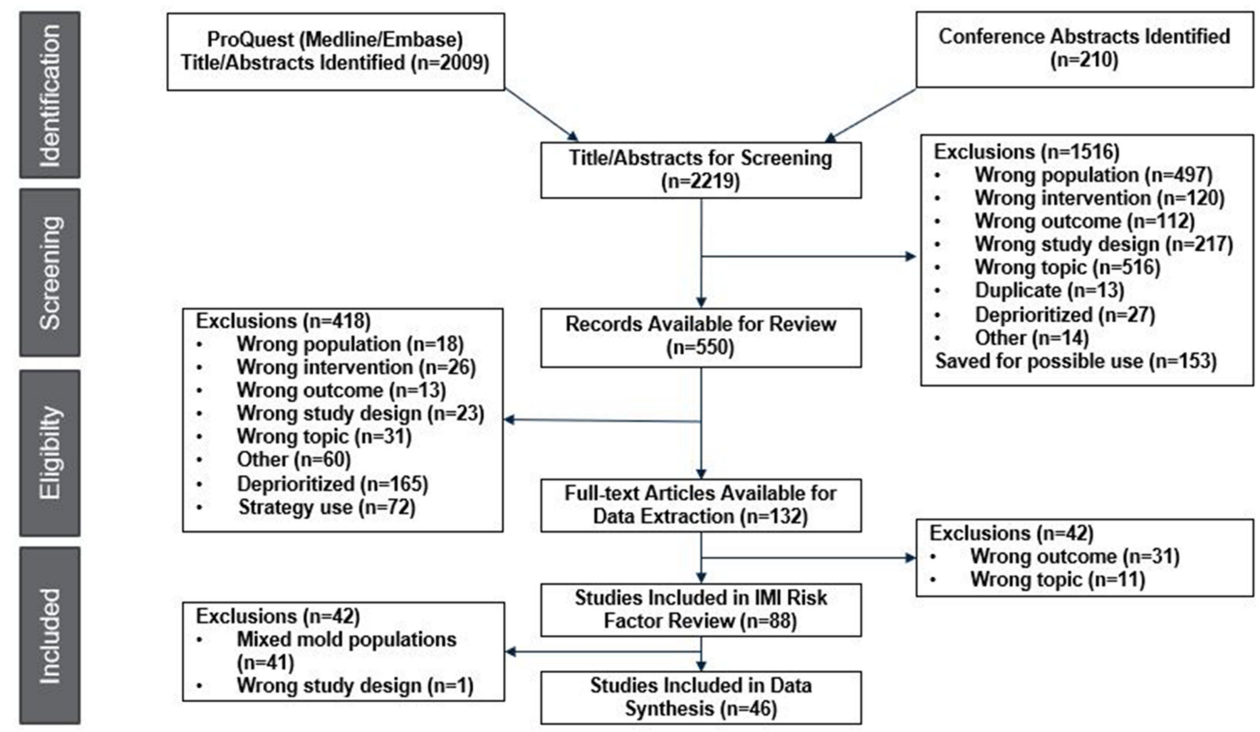

Figure I PRISMA Flow Diagram.

factors occurring in IA patients were: SOT (56.0\%), Heart Disease (51.0\%), Hematology Malignancy or Disease (46.0\%), and Liver Disease (38.0\%). The most frequently occurring comorbidities/risk factors occurring in IM patients in the literature were as follows: SOT (71.0\%), Immunocompromised (58.0\%), Hematology Malignancy or Disease (41.0\%), Diabetes (37.0\%), and Hematologic Transplant (35.0\%).

\section{Phase II Database Analysis Summary}

Of 101,340,454 patients in the Optum database, there were 5,730,144 (5.65\%) with diabetes; 9,095,448 (8.98\%) with lung disease; $125,381(0.12 \%)$ with SOT; and 962,428 $(0.95 \%)$ with HEM/ONC diagnoses (see Table 4). Within

Table 2 Comorbidities Proportions Across IMI Studies in the SLR

\begin{tabular}{|l|l|l|}
\hline $\begin{array}{l}\text { Comorbidity/Risk Factor } \\
\text { (46 Studies) }\end{array}$ & IA (n=35) & IM (n=I I) \\
\hline Healthy & 2 & 2 \\
Diabetes & 17 & 8 \\
Lung Disease & 10 & 2 \\
Hematology Malignancy or Disease & 17 & 7 \\
Solid Organ Transplant & 11 & 4 \\
Heart Disease & 9 & 0 \\
Liver Disease & 7 & 2 \\
Renal Impairment & 7 & 3 \\
Immuno-compromised & 8 & 4 \\
Hematologic transplant & 6 & 1 \\
Solid Malignancy & 7 & 3 \\
\hline
\end{tabular}

Abbreviations: IA, Invasive Aspergillosis; IM, Invasive Mucormycosis; IMI, Invasive mold infection; SLR, Systematic literature review. the diabetes cohort, $23,578(0.41 \%)$ were diagnosed with IA; 2,257 (0.04\%) with IM; and 117 (0.003\%) with both. Within the lung disease cohort, $42,939(0.71 \%)$ were diagnosed with IA; 3,264 (0.04\%) with IM; and $204(0.002 \%)$ with both. Within the HEM/ONC cohort, 10,638 (1.11\%) were diagnosed with IA; $1,133(0.12 \%)$ with IM; and 161 $(0.02 \%)$ with both. Within the SOT cohort, $2,536(2.02 \%)$ were diagnosed with IA; $144(0.11 \%)$ with IM; and 41 $(0.03 \%)$ with both.

Table 5 provides the mean post-index CCI scores for each risk cohort, overall and by mold infection group IA, IM, and co-infected cohorts. CCI scores were elevated in all mold infection groups compared to the overall cohort score (all $\mathrm{p}<0.0001)$. CCI scores were greatest in SOT

Table 3 Weighted Average Prevalence of Comorbidities Across IMI Patients in the SLR

\begin{tabular}{|l|l|l|}
\hline Comorbidity/Risk Factor (46 Studies) & IA & IM \\
\hline Healthy & $15.0 \%$ & $11.0 \%$ \\
Diabetes & $19.0 \%$ & $37.0 \%$ \\
Lung Disease & $46.0 \%$ & $12.0 \%$ \\
Hematology Malignancy or Disease & $48.0 \%$ & $41.0 \%$ \\
Solid Organ Transplant & $56.0 \%$ & $71.0 \%$ \\
Heart Disease & $51.0 \%$ & $\mathrm{NR}$ \\
Liver Disease & $38.0 \%$ & $3.0 \%$ \\
Renal Impairment & $23.0 \%$ & $18.0 \%$ \\
Immuno-compromised & $7.0 \%$ & $58.0 \%$ \\
Hematologic transplant & $4.0 \%$ & $35.0 \%$ \\
Solid Malignancy & $10.0 \%$ & $2.0 \%$ \\
\hline
\end{tabular}

Abbreviations: IA, Invasive Aspergillosis; IM, Invasive Mucormycosis; IMI, Invasive mold infection; NR, Not reported; SLR, Systematic literature review. 
Table 4 Rates of Invasive Mold Infections in Comorbidity and Risk Factor Groups

\begin{tabular}{|c|c|c|c|c|}
\hline Evaluation Group & $\mathbf{N}$ & Invasive Aspergillosis n (\%)* & Invasive Mucormycosis n (\%)* & Co-Infected n (\%)* \\
\hline Diabetes & $5,730,144$ & $23,578(0.41 \%)$ & $2257(0.04 \%)$ & $117(0.003 \%)$ \\
\hline Lung Disease & $9,095,448$ & $42,939(0.47 \%)$ & $3264(0.04 \%)$ & $204(0.002 \%)$ \\
\hline Cystic fibrosis & 19,030 & $1000(5.25 \%)$ & $12(0.06 \%)$ & $3(0.02 \%)$ \\
\hline Tuberculosis & 40,788 & $599(1.47 \%)$ & $30(0.07 \%)$ & $4(0.01 \%)$ \\
\hline COPD & $9,063,186$ & $42,719(0.47 \%)$ & $3255(0.04 \%)$ & $0(0.00 \%)$ \\
\hline HEM/ONC & 962,428 & $10,638(1.11 \%)$ & $1133(0.12 \%)$ & $161(0.02 \%)$ \\
\hline AML & 34,367 & 1562 (4.55\%) & 145 (0.42\%) & $66(0.19 \%)$ \\
\hline Lymphoma & 209,953 & $2242(1.07 \%)$ & $165(0.08 \%)$ & $40(0.02 \%)$ \\
\hline Leukemia & 126,982 & $2354(1.85 \%)$ & $208(0.16 \%)$ & $8 \mathrm{I}(0.06 \%)$ \\
\hline Other & 148,458 & II $33(0.76 \%)$ & $102(0.07 \%)$ & $25(0.02 \%)$ \\
\hline Stem Cell & 21,463 & $1065(4.96 \%)$ & $106(0.49 \%)$ & $42(0.20 \%)$ \\
\hline Neutropenia & 278,390 & 4299 (I.54\%) & $332(0.12 \%)$ & $113(0.04 \%)$ \\
\hline SOT & $|25,38|$ & $2536(2.02 \%)$ & $144(0.11 \%)$ & $4 \mathrm{I}(0.03 \%)$ \\
\hline Kidney transplant & 77,315 & 1097 (I.42\%) & $61(0.08 \%)$ & $14(0.02 \%)$ \\
\hline Heart transplant & 14,294 & $423(2.96 \%)$ & $33(0.23 \%)$ & $9(0.06 \%)$ \\
\hline Lung transplant & 9171 & 991 (10.81\%) & $39(0.43 \%)$ & $18(0.20 \%)$ \\
\hline Liver transplant & 32,456 & $760(2.34 \%)$ & $38(0.12 \%)$ & $7(0.02 \%)$ \\
\hline Pancreas transplant & 6268 & $155(2.47 \%)$ & $10(0.16 \%)$ & $3(0.05 \%)$ \\
\hline Heart and Lung transplant & 33 & $6(18.18 \%)$ & $0(0.00 \%)$ & $0(0.00 \%)$ \\
\hline Pancreas and Kidney transplant & 53 & $0(0.00 \%)$ & $0(0.00 \%)$ & $0(0.00 \%)$ \\
\hline Intestine transplant & 959 & $86(8.97 \%)$ & $8(0.83 \%)$ & $2(0.21 \%)$ \\
\hline Other transplant & 5606 & 132 (2.35\%) & $8(0.14 \%)$ & $3(0.05 \%)$ \\
\hline
\end{tabular}

Note: *Percent of evaluation cohort.

Abbreviations: AML, Acute Myeloid Leukemia; COPD, chronic obstructive pulmonary disorder; HEM/ONC, hematology and oncology; N, number of patients; SOT, Solid Organ Transplant.

Table 5 Mean Post-Index CCI Score in Diabetes, Lung Disease, SOT, and HEM/ONC Diagnosed Patients by IMI-Status

\begin{tabular}{|c|c|c|c|c|}
\hline Cohort & Entire Cohort & $\begin{array}{l}\text { Invasive Aspergillosis } \\
\text { Mean } \pm \text { SD } \\
\text { Diff }(95 \% \mathrm{Cl})^{\dagger} \\
\text { p-value }\end{array}$ & $\begin{array}{l}\text { Invasive Mucormycosis } \\
\text { Mean } \pm \text { SD } \\
\text { Diff }(95 \% \mathrm{Cl})^{\dagger} \\
\text { p-value }\end{array}$ & $\begin{array}{l}\text { Co-infected } \\
\text { Mean } \pm \text { SD } \\
\text { Diff }(95 \% \mathrm{Cl}) \dagger \\
\text { p-value }\end{array}$ \\
\hline Diabetes & $2.28 \pm 1.86 *$ & $\begin{array}{l}3.16 \pm 2.44 \\
0.88(0.86,0.9) \\
<0.0001\end{array}$ & $\begin{array}{l}2.55 \pm 2.05 \\
0.27(0.19,0.35) \\
<0.0001\end{array}$ & $\begin{array}{l}3.68 \pm 2.57 \\
I .40(1.13,1.67) \\
<0.0001\end{array}$ \\
\hline Lung Disease & $1.90 \pm 1.75^{*}$ & $\begin{array}{l}2.68 \pm 2.32 \\
0.78(0.76,0.80) \\
<0.0001\end{array}$ & $\begin{array}{l}2.98 \pm 2.18 \\
1.08(1.02,1.14) \\
<0.0001\end{array}$ & $\begin{array}{l}3.85 \pm 2.61 \\
1.95(I .7 I, 2.19) \\
<0.0001\end{array}$ \\
\hline HEM/ONC & $2.99 \pm 2.6 I^{*}$ & $\begin{array}{l}3.70 \pm 2.55 \\
0.7 \mid(0.66,0.76) \\
<0.0001\end{array}$ & $\begin{array}{l}3.52 \pm 2.11 \\
0.53(0.38,0.68) \\
<0.0001\end{array}$ & $\begin{array}{l}4.14 \pm 2.49 \\
I .15(0.75, I .55) \\
<0.000 ।\end{array}$ \\
\hline SOT & $3.26 \pm 2.28^{*}$ & $\begin{array}{l}4.00 \pm 2.83 \\
0.74(0.65,0.83) \\
<0.0001\end{array}$ & $\begin{array}{l}4.70 \pm 2.67 \\
1.44(1.07,1.81) \\
<0.0001\end{array}$ & $\begin{array}{l}4.88 \pm 2.79 \\
1.62(0.92,2.32) \\
<0.0001\end{array}$ \\
\hline
\end{tabular}

Notes: *Represents Mean CCl score for Total cohort, including IMI patients. †Difference relative to mean $\mathrm{CCl}$ score of Total cohort.

Abbreviations: $\mathrm{CCl}$, Charlson Comorbidity Index; Diff, Difference; HEM/ONC, hematology and oncology; IMI, Invasive mold infection; SD, Standard deviation; SOT, Solid Organ Transplant. 
patients (entire cohort: $3.26 \pm 2.28$; IA: $4.00 \pm 2.83$; IM: $4.70 \pm 2.67$; co-infected: $4.88 \pm 2.79$ ), and in those with co-infection across the cohorts (diabetes: $3.68 \pm 2.57$; lung disease $3.85 \pm 2.61 ; \mathrm{HEM} / \mathrm{ONC} 4.14 \pm 2.49$; SOT 4.88 $\pm 2.79)$.

In addition to the comorbidities used to calculate $\mathrm{CCI}$ scores, we found similarities in comorbid chronic conditions using top ICD-9/ICD-10 codes across cohorts. Diabetes, hypertension, hyperlipidemia, renal disease, chronic pulmonary disease (CPD), malignancy, and congestive heart failure (CHF) were found to be common and shared as comorbidities or risk factors between some or all of the four main cohorts, regardless of type of mold infection. The occurrence of classic metabolic comorbidities (diabetes, hypertension, hyperlipidemia) was higher in IM patients compared to IA patients (Table 6).

Demographic data are available in Appendix D for IA and IM infected patients in the diabetes, lung, HEM/ONC, and SOT comorbidity cohorts. Median ages of IA and IM patients in the comorbidity cohorts were diabetes (IA: 61 years; IM: 58 years), Lung (IA: 57 years; IM: 59 years), HEM/ONC (IA: 58 years; IM: 58 years), and SOT (IA: 56 years; IM: 54 years). Frequency of female sex in each group was diabetes (IA: 54.59\%; IM: 47.85\%), Lung (IA: $60.37 \%$; IM: 56.22\%), HEM/ONC (IA: 54.31\%; IM: 56.84\%), and SOT (IA: 41.05\%; IM: 42.36\%). Frequency of Caucasian race in each group was diabetes (IA: 77.97\%; IM: 81.61\%), Lung (IA: 83.07\%; IM: 85.97\%), HEM/ ONC (IA: 84.81\%; IM: 85.44\%), and SOT (IA: 78.94\%;
IM: 73.61\%). Frequency of Hispanic ethnicity in each group was diabetes (IA: 6.59\%; IM: 4.83\%), Lung (IA: 3.61\%; IM: 2.60\%), HEM/ONC (IA: 4.10\%; IM: 4.59\%), and SOT (IA: 7.97\%; IM: 12.50\%).

\section{Discussion}

The current study used mixed methods, combining both an SLR and a database analysis to describe patient comorbidity and risk factors associated with IA or IM, or other mold infections. The systematic literature review confirms and highlights current understanding of mold infection risk factors. The results of the database analysis further our understanding of IA, IM, and co-infection in patients diagnosed with diabetes, lung disease, hematologic malignancy or disease, and solid organ transplants while revealing additional considerations.

A number of shared chronic disease comorbidities were observed across some or all of the cohorts using the Optum dataset cohort. Essential (primary) hypertension was almost twice as frequent in IM patients compared to IA patients. However, literature on heart disease risk factors in IM were lacking $(n=0)$ and therefore not considered for our cohorts although some studies were found for IA $(n=9)$. Hyperlipidemia, long-term (current) use of other medications, and diabetes were additional notable comorbidities shared within our four cohorts, regardless of the type of mold infection. Larger percentages of IM patients had metabolic comorbidities (hypertension, hyperlipidemia, diabetes) compared to IA patients, thus

Table 6 Frequencies of Common Metabolic Comorbidities by Cohort and Type of Infection

\begin{tabular}{|l|c|c|c|}
\hline Cohort & Total (\%)* & Invasive Aspergillosis (\%)* & Invasive Mucormycosis (\%)* \\
\hline Diabetes & & & $38-55$ \\
Essential hypertension & $28-45$ & 25 & $42-72$ \\
Hyperlipidemia & $18-36$ & & $50-62$ \\
\hline Lung Disease & & $27-36$ & $32-36$ \\
Essential hypertension & $12-25$ & 18 & $41-50$ \\
Hyperlipidemia & 11 & $28-34$ & $28-29$ \\
\hline HEM/ONC & 25 & 16 & $28-33$ \\
Essential hypertension & $15-18$ & & 48 \\
Hyperlipidemia & & NR & 30 \\
\hline SOT & 12 & 38 & 22 \\
Diabetes & 28 & & \\
Essential hypertension & $15-22$ & & \\
Hyperlipidemia & & & \\
\hline
\end{tabular}

Notes: *Represents frequencies of top ICD-9/ICD-10 codes reported for patient cohort $>20 \%$; ranges reflect differences in codes.

Abbreviations: HEM/ONC, hematology and oncology; SOT, Solid Organ Transplant; NR, Not reported. 
suggesting their importance for consideration when suspecting mold, specifically Mucormycosis. HIV/AIDS and dementia, diagnoses included within the CCI, were consistently the least occurring comorbidities across the cohorts. Although HIV/AIDS have been documented as a significant comorbidity in immunocompromised patients and liver failure patients, ${ }^{30,31}$ they may be underrepresented in the Optum commercial dataset. Finally, CCI cohort demonstrated higher comorbidity burdens for patients developing invasive mold infections, indicating higher mortality and/or higher resource use at the outset.

Overall, the findings from the database were consistent with the literature, but allowed further insight into the comorbidities, particularly the added burden of more frequent chronic metabolic conditions in patients with Mucormycosis. The chronic disease metabolic comorbidities noted in our database analysis for IA and IM are also similar risk factors in patients admitted to intensive care (ICU) in the era of COVID-19. ${ }^{7}$ It is expected that some patients with COVID-19 will require treatment for invasive fungal infections related to prolonged ICU stays and treatments, even without the presence of classic host criteria. Expanded knowledge of common comorbidities occurring with invasive mold infections may be useful in earlier suspicion, diagnosis, and treatment, especially in non-traditional hosts and those with emerging risk factors. Increasing awareness, given the challenging diagnostic work-up, is important to appropriately address possible mold infections early in order for clinicians to maintain focus on treating patients' underlying illnesses.

Furthermore, knowledge of which risk factors and comorbidities are more likely to be seen with either IA or IM can help healthcare providers best plan antifungal treatment strategies that are optimal to unique patient characteristics. For example, type 2 diabetes patients are at higher risk of IA and IM, and are more likely to be comorbid with hyperlipidemia, hypertension, and obesity, making dosing with some antifungals difficult. For obese critically ill patients, weight-based dosing is challenging as antifungal pharmacokinetics vary, specifically the volume of distribution. This could impact the efficacy and toxicity of treatment ${ }^{32}$ like voriconazole and L-AMB, which both involve weight-based dosing ${ }^{33,34}$ or posaconazole, which may have lower plasma drug concentrations in patients $>120 \mathrm{~kg} .{ }^{35}$ In these patients, treatment options without weight-based dosing requirements and without trough level concentration difference between obese and nonobese patients as well as minimizing drug-drug interactions would be preferable and make suboptimal dosing less likely. ${ }^{36-38}$

In terms of guidelines, the Infectious Diseases Society of America recommends voriconazole with amphotericin B formulations as first-line therapy for IA and isavuconazole as primary alternative with echinocandins in intolerant or refractory cases. ${ }^{39}$ For IM, the European Society for Clinical Microbiology and Infectious Diseases and European Confederation of Medical Mycology recommend amphotericin $\mathrm{B}$, isavuconazole or posaconazole with adjunctive surgical intervention when possible. ${ }^{40} \mathrm{~A}$ few studies reported the use of antifungal agents for proven/possible mucormycosis. ${ }^{41,42}$ One study was an SLR, and the other two were retrospective studies. In the multicenter retrospective study of 74 cases from Australia, an amphotericin B formulation (predominantly liposomal amphotericin B) was the mainstay of antifungal therapy (62/64 cases), with amphotericin B-based combination therapy administered in 11 cases $(17.7 \%)$, including caspofungin $(n=5)$, posaconazole $(n=5)$ and terbinafine $(n=1)$. Posaconazole solution was used as step-down therapy in all cases where the patient was alive following initial "induction antifungal therapy". ${ }^{41}$ In a Spanish retrospective study, 17 mucormycosis cases from a large hospital where all patients received antifungal treatment with 1 or more antifungal agents, mainly liposomal amphotericin B (18/19) with one patient receiving caspofungin and voriconazole. ${ }^{42}$ However, both studies were conducted prior to isavuconazole gaining market access.

There are some limitations to this mixed-methods study. We used an SLR to focus on patient risk factors alone, while future reviews could include clinical and economic outcomes of treatments. Generalizability to diverse patients may be limited as the majority of the mold infection data in the SLR came from studies in North America and Europe and the patient demographics from the US database analysis (median age range 54-61, high-frequency Caucasian, low frequency Hispanic ethnicity) were representative of the Optum database population but generally uniform. Another key limitation is that a claims database was used that did not have full clinical details around antifungals and other interventions/procedures (timing, dosing). As such, analyses were not performed to compare treatment patterns between different patient cohorts.

\section{Conclusions}

Our findings from the SLR and database analysis confirms and expands the current evidence base regarding risk factors and comorbidities for invasive mold infections. 
Knowledge of comorbidities and risk factors may improve suspicion of mold, diagnosis, and early treatment. Given the various patient profiles that may be at risk for IMI, understanding which antifungal treatments are best suited to treat mold infections in traditional high-risk (SOT, hematologic malignancy) and non-traditional hosts (diabetes, lung disease, heart disease, etc.) is important. Such research would inform best practices for treatment as well as identify current gaps in treatment options for various patient risk groups. Additional research is needed in Africa-Middle East and Asia-Pacific regions to determine if the same risk factors and comorbidities drive invasive mold infections outside of Europe and North America.

\section{Declaration of Funding}

Research funding was provided by Pfizer Inc.

\section{Disclosure}

AHS, BP, MB, JS, RC, JAA are employees of Pfizer. SM and JS are employees of OPEN Health. JS also holds OPEN Health stock. OPEN Health recieved research funding from Pfizer. The authors report no other conflicts of interest in this work.

\section{References}

1. Harpaz R, Dahl R, Dooling K. The Prevalence of Immunocompromised Adults: United States, 2013. Oxford University Press; 2016.

2. Krcmery V, Kunova E, Jesenska Z, et al. Invasive mold infections in cancer patients: 5 years' experience withAspergillus, Mucor, Fusarium andAcremonium infections. Support Care Cancer. 1996;4(1):39-45. doi:10.1007/BF01769874

3. Kriengkauykiat J, Ito JI, Dadwal S. Epidemiology and treatment approaches in management of invasive fungal infections. Clin Epidemiol. 2011;3(1):175-191. doi:10.2147/CLEP.S12502

4. Omrani AS, Almaghrabi RS. Complications of hematopoietic stem transplantation: fungal infections. Hematol Oncol Stem Cell Ther. 2017;10(4):239-244. doi:10.1016/j.hemonc.2017.05.013

5. Enoch DA, Yang H, Aliyu SH, Micallef C. The changing epidemiology of invasive fungal infections. Methods Mol Biol. 2017;1508:17-65. doi:10.1007/978-1-4939-6515-1 2

6. Schauwvlieghe A, Rijnders BJA, Philips N, et al. Invasive aspergillosis in patients admitted to the intensive care unit with severe influenza: a retrospective cohort study. Lancet Respir Med. 2018;6(10):782-792. doi:10.1016/s2213-2600(18)30274-1

7. Apostolopoulou A, Esquer Garrigos Z, Vijayvargiya P, Lerner AH, Farmakiotis D. Invasive pulmonary aspergillosis in patients with SARS-CoV-2 infection: a systematic Review of the literature. Diagnostics (Basel). 2020;10(10):807. doi:10.3390/diagnostics10 100807

8. Anastasopoulou A, DiPippo AJ, Kontoyiannis DP. Non-Aspergillus invasive mould infections in patients treated with ibrutinib. Mycoses. 2020;63(8):787-793. doi:10.1111/myc. 13120

9. Vallabhaneni S, Benedict K, Derado G, Mody RK. Trends in hospitalizations related to invasive aspergillosis and mucormycosis in the United States, 2000-2013. Open Forum Infect Dis. 2017;4(1). doi:10.1093/ofid/ofw268
10. Morgan J, Wannemuehler KA, Marr KA, et al. Incidence of invasive aspergillosis following hematopoietic stem cell and solid organ transplantation: interim results of a prospective multicenter surveillance program. Med Mycol. 2005;43(Suppl s1):S49-58. doi:10.1080/ 13693780400020113

11. Sinkó J, Csomor J, Nikolova R, et al. Invasive fungal disease in allogeneic hematopoietic stem cell transplant recipients: an autopsy-driven survey. Transpl Infect Dis. 2008;10(2):106-109. doi:10.1111/j.1399-3062.2007.00264.x

12. Roden MM, Zaoutis TE, Buchanan WL, et al. Epidemiology and outcome of zygomycosis: a review of 929 reported cases. Clin Infect Dis. 2005;41(5):634-653. doi:10.1086/432579

13. Skiada A, Pagano L, Groll A, et al. Zygomycosis in Europe: analysis of 230 cases accrued by the registry of the European Confederation of Medical Mycology (ECMM) working group on zygomycosis between 2005 and 2007. Clin Microbiol Infect. 2011;17 (12):1859-1867. doi:10.1111/j.1469-0691.2010.03456.x

14. Bassetti M, Bouza E. Invasive mould infections in the ICU setting: complexities and solutions. $J$ Antimicrob Chemother. 2017;72 (suppl_1):i39-i47. doi:10.1093/jac/dkx032

15. Baddley JW. Clinical risk factors for invasive aspergillosis. Med Mycol. 2011;49(S1):S7-S12. doi:10.3109/13693786.2010.505204

16. Maertens J, Verhaegen J, Lagrou K, Van Eldere J, Boogaerts M. Screening for circulating galactomannan as a noninvasive diagnostic tool for invasive aspergillosis in prolonged neutropenic patients and stem cell transplantation recipients: a prospective validation. Blood. 2001;97(6):1604-1610. doi:10.1182/blood.v97.6.1604

17. Latgé JP. Aspergillus fumigatus and aspergillosis. Clin Microbiol Rev. 1999;12(2):310-350.

18. Kontoyiannis DP, Sumoza D, Tarrand J, Bodey GP, Storey R, Raad II. Significance of aspergillemia in patients with cancer: a 10-year study. Clin Infect Dis. 2000;31(1):188-189. doi:10.1086/ 313918

19. von Lilienfeld-toal M, Wagener J, Einsele H, Cornely OA, Kurzai O. Invasive fungal infection. Dtsch Arztebl Int. 2019;116(16):271-278. doi:10.3238/arztebl.2019.0271

20. Dignani MC. Epidemiology of invasive fungal diseases on the basis of autopsy reports. F1000Prime Rep. 2014;6:81. doi:10.12703/p6-81

21. Winters B, Custer J, Galvagno SM Jr., et al. Diagnostic errors in the intensive care unit: a systematic review of autopsy studies. BMJ Qual Saf. 2012;21(11):894-902. doi:10.1136/bmjqs-2012-000803

22. Donnelley MA, Zhu ES, Thompson GR 3rd. Isavuconazole in the treatment of invasive aspergillosis and mucormycosis infections. Infect Drug Resist. 2016;9:79-86. doi:10.2147/IDR.S81416

23. Ashley ESD, Lewis R, Lewis JS, Martin C, Andes D. Pharmacology of Systemic Antifungal Agents. Clin Infect Dis. 2006;43 (Supplement_1):S28-S39. doi:10.1086/504492

24. Gallis HA, Drew RH, Pickard WW. Amphotericin B: 30 Years of Clinical Experience. Rev Infect Dis. 1990;12(2):308-329. doi:10.1093/clinids/12.2.308

25. Label. CFP. Available from: https://www.accessdata.fda.gov/drug satfda_docs/label/2015/207500Orig1s000lbl.pdf. Accessed November 20, 2020.

26. Hutton B, Salanti G, Caldwell DM, et al. The PRISMA extension statement for reporting of systematic reviews incorporating network meta-analyses of health care interventions: checklist and explanations. Ann Intern Med. 2015;162(11):777-784. doi:10.7326/ m14-2385

27. Charlson ME, Pompei P, Ales KL, MacKenzie CR. A new method of classifying prognostic comorbidity in longitudinal studies: development and validation. $J$ Chronic Dis. 1987;40(5):373-383. doi:10.1016/0021-9681(87)90171-8

28. Quan H, Sundararajan V, Halfon P, et al. Coding algorithms for defining comorbidities in ICD-9-CM and ICD-10 administrative data. Med Care. 2005;43(11):1130-1139. doi:10.1097/01. mlr.0000182534.19832.83 
29. D'Hoore W, Sicotte C, Tilquin C. Risk adjustment in outcome assessment: the Charlson comorbidity index. Methods Inf Med. 1993;32 (05):382-387. doi:10.1055/s-0038-1634956

30. Wessolossky M, Welch VL, Sen A, Babu TM, Luke DR. Invasive Aspergillus infections in hospitalized patients with chronic lung disease. Infect Drug Resist. 2013;6:33-39. doi:10.2147/IDR.S43069

31. Zhang XY, Yang M, Hu J, Zhao H, Li L. Epidemiology of invasive pulmonary aspergillosis in patients with liver failure: clinical presentation, risk factors, and outcomes. J Int Med Res. 2018;46 (2):819-827. doi:10.1177/0300060517729907

32. Amsden JR, Slain D. Dosing antifungals in obesity: a literature review. Curr Fungal Infect Rep. 2019;13(1):21-32. doi:10.1007/ s12281-019-0335-3

33. Pfizer. VFEND (voriconazole) Prescribing Information. New York, NY. 2019.

34. Astellas Pharma US I. AmBisome (amphotericin B) liposome for injection - Prescribing Information. Northbrook, IL. 2019.

35. Co M. Noxafil (posaconazole) Prescribing Information. Whitehouse Station, NJ 2020.

36. Astellas Pharma US I. CRESEMBA (isavuconazonium sulfate) Prescribing Information. Northbrook, IL 2019.

37. Desai A, Kovanda L, Andes DR, et al. No Dose Adjustment Necessary for Isavuconazole in Obese Patients. Oxford University Press; 2016.
38. Desai AVK, Kovanda L, Hope WW, et al. Exposure-response relationships for isavuconazole in patients with invasive aspergillosis and other filamentous fungi. Antimicrob Agents Chemother. 2017;61 (12):12. doi:10.1128/AAC.01034-17

39. Walsh TJ, Anaissie EJ, Denning DW, et al. Treatment of aspergillosis: clinical practice guidelines of the infectious diseases society of America. Clin Infect Dis. 2008;46(3):327-360. doi:10.1086/525258

40. Cornely OA, Alastruey-Izquierdo A, Arenz D, et al. Global guideline for the diagnosis and management of mucormycosis: an initiative of the European confederation of medical mycology in cooperation with the mycoses study group education and research consortium. Lancet Infect Dis. 2019;19(12):e405-e421. doi:10.1016/s1473-3099(19) 30312-3

41. Kennedy KJD, Slavin MA, van Hal SJ, et al. Mucormycosis in Australia: contemporary epidemiology and outcomes. Clin Microbiol Infect. 2016;22(9):775-781. doi:10.1016/j.cmi.2016.0 1.005

42. Guinea J, Escribano P, Vena A, et al. Increasing incidence of mucormycosis in a large Spanish hospital from 2007 to 2015: epidemiology and microbiological characterization of the isolates. PLoS One. 2017;12(6):e0179136. doi:10.1371/journal.pone.0179136
ClinicoEconomics and Outcomes Research

\section{Publish your work in this journal}

ClinicoEconomics and Outcomes Research is an international, peerreviewed open-access journal focusing on Health Technology Assessment, Pharmacoeconomics and Outcomes Research in the areas of diagnosis, medical devices, and clinical, surgical and pharmacological intervention. The economic impact of health policy and health systems

\section{Dovepress}

organization also constitute important areas of coverage. The manuscript management system is completely online and includes a very quick and fair peer-review system, which is all easy to use. Visit http://www.dovepress.com/testimonials.php to read real quotes from published authors. 